\title{
Natural forest regeneration in abandoned sugarcane fields in northeastern Brazil: floristic changes
}

\author{
Ladivania Medeiros do Nascimento ${ }^{1,5}$, Everardo Valadares de Sá Barretto Sampaio ${ }^{2}$, \\ Maria Jesus Nogueira Rodal ${ }^{3}$, Suzene Izídio da Silva ${ }^{3}$ \& Ana Carolina Borges Lins e Silva ${ }^{4}$ \\ ${ }^{1}$ Jardim Botânico do Recife, Secretaria de Meio Ambiente, Prefeitura do Recife, Rod. BR 232, Km 7, \\ Coqueiral, CEP 54240-450, Recife, PE, Brasil \\ ${ }^{2}$ Departamento de Energia Nuclear, Universidade Federal de Pernambuco - UFPE, Av. Professor Moraes \\ Rego, 1235, Cidade Universitária, CEP 50670-901, Recife, PE, Brasil. www.ufpe.br \\ ${ }^{3}$ Departamento de Biologia, Área de Botânica, Universidade Federal Rural de Pernambuco - UFRPE, \\ Rua Dom Manoel de Medeiros, s/n, Dois Irmãos, CEP 52171-900, Recife, PE, Brasil. www.ufrpe.br \\ ${ }^{4}$ Departamento de Biologia, Área de Ecologia, Universidade Federal Rural de Pernambuco - UFRPE, \\ Rua Dom Manoel de Medeiros, s/n, Dois Irmãos, CEP 52171-900, Recife, PE, Brasil. www.ufrpe.br \\ ${ }^{5}$ Corresponding author: Ladivania Medeiros do Nascimento, e-mail: ladivania@hotmail.com
}

NASCIMENTO, L.M., SAMPAIO, E.V.S.B., RODAL, M.J.N., SILVA, S.I. \& LINS e SILVA, A.C.B. Natural forest regeneration in abandoned sugarcane fields in northeastern Brazil: floristic changes. Biota Neotrop. 12(4): http://www.biotaneotropica.org.br/v12n4/en/abstract?article+bn02012042012

Abstract: Surveys were undertaken to examine the floristic changes during secondary succession in three areas of 12 and three of 20-year-old secondary forests in Pernambuco State, Brazil. Two hundred and six species were identified, with 136 being found in the 12-year-old secondary forest and 161 species in the 20-year-old forest. Fabaceae and Myrtaceae were the most important families, increasing in species numbers with regeneration age. Of the 216 species, 115 were trees, 48 shrubs, 16 herbaceous plants, and 24 woody lianas, without significant differences between the two regeneration site ages. NMDS analysis revealed a formation of two floristic groups, distinguishing secondary and mature forests, with a further division within secondary forests in accordance with the time since abandonment. Similarity analysis ANOSIM confirmed the significance of the groups, which had floristic composition significant distinct $(\mathrm{R}=0.96)$ and $63 \%$ of dissimilarity (SIMPER). However, the sharing of 68 arboreal species between the secondary and mature forests suggests a floristic convergence. DCA analysis of the arboreal component as well as the other plant habits suggested that the separation of the subgroups is correlated with physical and chemical variables of the soils. All of these results indicate that, within the chronosequence analyzed, the velocity and direction of the floristic composition during secondary succession was influenced not only by the time of their abandonment, but also by a wide range of environmental variables.

Keywords: secondary forests, floristic, secondary succession.

NASCIMENTO, L.M., SAMPAIO, E.V.S.B., RODAL, M.J.N., SILVA, S.I. \& LINS e SILVA, A.C.B. Regeneração natural de mata em áreas de cana abandonadas no nordeste do Brasil: mudanças florísticas. Biota Neotrop. 12(4): http://www.biotaneotropica.org.br/v12n4/pt/abstract?article+bn02012042012

Resumo: Com objetivo de detectar mudanças florísticas ao longo da sucessão secundária e subsidiar futuros projetos de recuperação florestal foi realizado levantamento florístico de seis áreas de floresta secundária (capoeira) de 12 e 20 anos em Pernambuco. Foram registradas 206 espécies, sendo 136 nas capoeiras de 12 anos e 161 nas de 20 anos. Fabaceae e Myrtaceae foram as famílias mais importantes, aumentando no número de espécies com a idade de regeneração. Do total de espécies, 115 foram árvores, 48 arbustos, 16 herbáceas e 24 trepadeiras, sem diferença significativa por idade de regeneração. Análise de NMDS indicou a separação dos grupos florísticos das florestas maduras e das capoeiras, assim como a formação de subgrupos de capoeiras (SF1-2-3 e SF4-5-6) com idade de regeneração distinta. A análise de similaridade ANOSIM mostrou que os grupos formados apresentaram composição florística significativamente distintas $(\mathrm{R}=0.96)$ e $63 \%$ de dissimilaridade (SIMPER). Entretanto, a presença nas capoeiras de 67 espécies em comum com as florestas maduras indicam uma tendência de convergência florística. A análise de DCA do componente arbóreo e dos outros hábitos sugere que a separação dos subgrupos por idade estaria correlacionada com variáveis edáficas físicas e químicas. Todos esses resultados indicam que, numa análise de cronossequência, não apenas o tempo de abandono, mas todas as variáveis ambientais influenciam a velocidade e direção de formação da composição florística durante a sucessão secundária.

Palavras-chave: capoeira, flora vascular, sucessão secundária. 


\section{Introduction}

The degradation and destruction of natural habitats figure among the principal modern threats to biodiversity (Primack 2008). With the acceleration of the conversion of forests into pasture lands and agricultural fields in recent decades in Latin America (Geist \& Lambin 2001) it has come to the point that mature forests altered by anthropogenic actions and regenerating secondary forests now compose approximately half of all remaining forest areas in the tropics (International... 2002). On the other hand, large areas of formerly cleared lands have also been abandoned and are evolving into secondary forests (Wright 2005).

Fragments of mature and secondary forests can be found in many landscapes otherwise dominated by agriculture or pasture lands. This situation can be seen, for example, in the Brazilian Atlantic Forest biome that has suffered negative impacts from a number of economic cycles, resulting in significant landscape modifications and ecosystem destruction (Dean 2002). The conservation of the Atlantic Forest and its biodiversity now presents a significant challenge to conservation efforts, principally due to its advanced state of forest substitution, and depends on the protection of the remaining fragments of mature forest (Tabarelli \& Gascon 2005) and the correct management of regenerating forests after abandonment (Dent \& Wright 2009). The Atlantic Forest now persists on only between $11 \%$ and $16 \%$ of its original land area coverage, and even these remnants are extremely fragmented (Ribeiro et al. 2009). This once extensive forest is currently composed of just a few well-preserved mature forest sites with numerous areas of varying ages with diverse use-histories undergoing secondary succession, and these are often surrounded by matrixes of cultivated areas and pasturelands (Ranta et al. 1998, Trindade et al. 2008).

A number of workers have pointed out the importance of secondary forests: as biodiversity reservoirs within fragmented landscapes (Chazdon 1998, 2003, Letcher \& Chazdon 2009); as sources of sustenance for wildlife (Parry et al. 2007, Herrera-Montes \& Brokaw 2010), providing sites for conservation of rare and endemic species (Liebsch et al. 2008) and wood and non-wood products (Chazdon \& Coe 1999); in the accumulation of biomass (Gehring et al. 2004, Grace 2004, Feldpausch et al. 2007); for controlling carbon emissions (Feldpausch et al. 2005); and for diminishing pressure on natural habitats (Wright \& Muller-Landau 2006).

The structural attributes of tropical forests such as density, biomass, richness, diversity, and composition can slowly recover after significant natural or anthropogenic disturbances (Brown \& Lugo 1990, Guariguata \& Ostertag 2001, Chazdon 2003, Finegan \& Nasi 2004), but an important question in terms of our understanding of successional processes of secondary forests is if their floristic composition also tends to converge towards that of nearby mature forests or if they maintain their differences. According to Chazdon et al. (2009), convergence seems to be related to the level of anthropogenic disturbance, the duration of the time that the area was used, and the regional landscape context. The interrelations of these parameters make the outcomes of succession less predictable, and measurable differences in the floristic compositions of secondary forests may persist even centuries after they have been abandoned and left to recuperate (Finegan 1996, Aide et al. 2000, Denslow \& Guzman 2000, Calvo et al. 2002, Ribas et al. 2003).

Previous researchers (refer to a review by Guariguata \& Ostertag 2001) have demonstrated that a number of factors influence the recuperation of the floristic composition after disturbances, although many questions still remain unanswered due, in large part, to the idiosyncratic, non-directional, and largely unpredictable processes of succession in tropical forests (Letcher \& Chazdon 2009).
The present work analyzed the floristic composition of 12 and 20-year-old secondary forests (known in northeastern Brazil as capoeiras) growing within a landscape dominated by sugarcane plantations and compared them with mature forest fragments of various sizes and showing varying degrees of disturbance, with the intention of addressing two basic questions: 1) Do 12 and 20-year-old secondary forest tend to floristically converge to resemble neighboring mature forests? 2) Is their time of abandonment/recuperation the principal factor determining this conversion process?

\section{Materials and Methods}

Weekly collections were made between June/2006 and July/2009 in six secondary forest fragments (capoeiras) belonging to the Usina São José (USJ) sugar refinery in Igarassu, Pernambuco State (PE), Brazil. The fragments were located between the geographical coordinates $07^{\circ} 41^{\prime} 04.9^{\prime \prime}$ and $07^{\circ} 54^{\prime} 41.6^{\prime \prime} \mathrm{S}$ and $34^{\circ} 54^{\prime} 17.6^{\prime \prime}$ and $35^{\circ} 05^{\prime} 07.2^{\prime} \mathrm{W}$, within a total area of approximately $280 \mathrm{~km}^{2}$ (Figure 1); $88 \%$ of that area was occupied by a monoculture of sugarcane (Trindade et al. 2008). The six forest fragments had sizes that varied from 5 to $11 \mathrm{ha}$, and they were up to $300 \mathrm{~m}$ from mature forest fragments, the latter being considered mature in not having suffered any clear-cutting for at least 60 years.

The local climate is of the type As' (Köppen 1936), characterized as hot and humid, with an average annual rainfall rate of $1687 \mathrm{~mm}$, average temperature of $24.9^{\circ} \mathrm{C}$, and a dry season lasting more than three months (Schessl et al. 2008). The predominant geological formation in the area is the Barreiras Formation of the plioPleistocene (the most extensive geologic formation in this region of the coast) that comprises nonconsolidated sandy-clay sediments of continental origin (Companhia... 2003). The landscape is dominated by coastal plateaus 40 to $160 \mathrm{~m}$ above sea level that are cut by deep and narrow valleys whose sides have inclinations greater than $30 \%$ (Companhia... 2003). The regional vegetation is classified as Dense Ombrophilous Lowland Forest (Veloso et al. 1991).

Site selections considered the approximate ages of the secondary forest (capoeira) remnants based on the vegetational characteristics captured in aerial photographic sequences (CONDEPE/FIDEM) covering the decades of 1960,1970, and 1980 (at a scale of $1: 30,000$ ), as well as satellite images acquired in 2005 and disturbance histories from the 1990s obtained from interviews with long-time residents in the area.

Of the six secondary forest sites examined, three had been undergoing natural regeneration for approximately 12 years (sites 1, 2 and 6) and three had regenerated for 20 years (sites 3, 4 and 5) after suspending sugarcane cultivation during the 1980s. When they were selected in 2006, the six areas had shrub/arboreal canopy physiognomies varying from 6 to $18 \mathrm{~m}$ in height.

Botanical material was collected, using traditional techniques (Mori et al. 1989), crisscrossing the fragment in random walks (Filgueiras et al. 1994) and also examining each plant within one hundred and eighty $10 \times 10 \mathrm{~m}$ plots installed in the six fragments $(30$ plots in each fragment). In each fragment, the whole fragment was surveyed, including edge and interior areas. Growth habits examined were arboreal (diameter at breast height, $\mathrm{DBH} \geq 15 \mathrm{~cm}$ ), shrub/ subshrubs and terrestrial herbs $(\mathrm{DBH}<15 \mathrm{~cm})$, epiphytic herbs and woody lianas, all with reproductive parts, whether flowers or fruits. Seedlings or saplings of arboreal species were not included.

Soil samples were collected in each plot from the first 20 centimeters below the surface and analyzed in terms of their soil texture (sand, silt, and clay), $\mathrm{pH}$ in water, $\mathrm{P}, \mathrm{K}^{+}, \mathrm{Ca}^{2+}, \mathrm{Mg}^{2+}$, and extractable $\mathrm{Al}^{3+}$, according to protocols described in the Manual of Soil Analysis Methods (Embrapa 1997). 


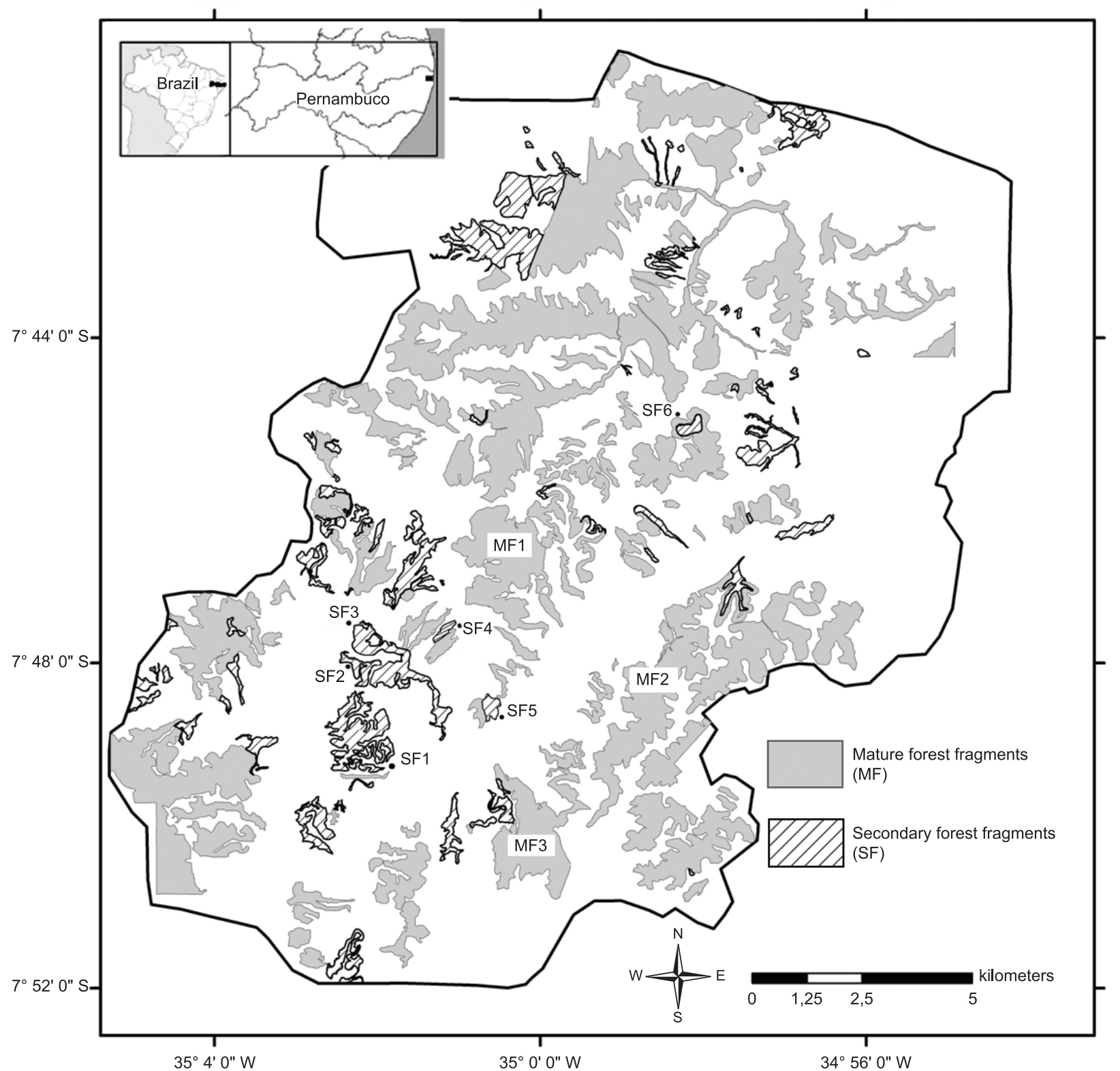

Figure 1. Location of 12-year (SF1, SF2 and SF6) and 20-year secondary forests (SF3, SF4 and SF5) and of mature forests (MF1=Silva (2004), MF2 = Rocha et al. (2008), MF3 = Silva et al. (2008)) in Usina São José, Igarassu municipality, Pernambuco state, Brazil.

Reference collections were incorporated into the Geraldo Mariz Herbarium (UFP), with duplicates to the Dárdano de Andrade Lima Herbarium (IPA). Species identifications were made by specialists at different Brazilian institutions and by comparisons with collections deposited at the Professor Vasconcelos Sobrinho (PEUFR) and IPA herbaria.

A species list was prepared, listed according to family, with information about plant habits, localities of occurrence, and their herbarium collection numbers. The classification of the angiosperm families followed the recommendations of the APG III (Angiosperm... 2009), and those of the pteridophytes followed Smith \& Wolf (2006), with modifications as presented by Smith et al. (2008). The authors' names and scientific names were confirmed using The International Plant Names Index (www.ipni.org) database.

Statistical differences in the soils were tested using one-way ANOVA (Stat Soft 2001). Values were transformed before analysis if they did not exhibit normal distribution or variance homogeneity, following Sokal \& Rohlf (1995).

The G test (Sokal \& Rohlf 1995) was used to identify changes in the species richness of each plant habit between the 12 and 20 -yearold secondary forests.

The floristic compositions of the arboreal component of mature forest fragments in the study area were obtained from the works of Silva (2004), Rocha et al. (2008), and Silva et al. (2008), which included all trees with $\mathrm{DBH} \geq 15 \mathrm{~cm}$, similarly to the present study. Four types of analyses were performed to examine the arboreal habit data (NMDS, ANOSIM, SIMPER and DCA-Detrended Correspondence Analysis), utilizing the PAST 2.01 (Hammer et al. 2001) and PC-ORD version 4.0 software program (McCune \& Mefford 1999).

The NMDS analysis (Non-metric Multi-dimensional Scaling) was used to analyze the degree of floristic difference between the two secondary forest ages (12 and 20-year-old), and between those 
and nearby mature forests. To that end, a binary matrix (presence/ absence) of the secondary forests ( 115 species) and the mature forests (171 spp.) was constructed. Infra-specific taxa or those without precise identification to the species level (listed as "sp.", "cf." or "aff."), which occurred in at least two areas, were excluded from analysis, resulting in a matrix of 136 species. In the analysis, the Jaccard (J) index coefficient was applied to generate a graph (Legendre \& Legendre 1998).

The non-parametric analyzis ANOSIM (Clarke 1993) was also performed, com 10,000 permutations, aiming at confirming the significance of the groups formed by the NMDS analysis. This method generates a global R-statistic, which is a measure of the distance between groups. An R-value close to 1 indicates strongly dissimilar assemblages, while an R-value close to zero indicates that assemblages are scarcely distinguishable (Clarke 1993). These $\mathrm{R}$-values were used to compare floristic assemblages between secondary vegetation ages. Where ANOSIM revealed significant differences between groups, Similarity percentages (SIMPER) analysis was used to identify those species that contributed most to the observed assemblage distinction (Clarke 1993). Cumulative contributions were cut arbitrarily at $50 \%$. Species with the highest dissimilarity to standard deviation ratios were identified as good discriminators for each comparison (Clarke 1993).
A third analysis (DCA) was applied to determine correlations between the species distributions and environmental variables. Data concerning the presence/absence (0-1) of the arboreal species (115 spp.) and the set of other habits (shrubs, herbs, and woody lianas $=91$ spp.) were used to form two primary matrices. A categorical variable matrix was created based of the average values of the chemical $(\mathrm{pH}$, $\mathrm{P}, \mathrm{K}^{+}, \mathrm{Ca}^{2+}, \mathrm{Mg}^{2+}, \mathrm{Al}^{3+}$ ) and physical (percentages of sand, clay, and silt) analyses of the soils and with the time of vegetation recuperation (12 and 20 years). The Pearson and Kendall correlation was applied to evaluate the representativeness of the variables on the axes; $r$ values $\geq 0.70$ were considered high (Cohen 1988, Dancey \& Reidy 2006).

\section{Results}

Sixty-six families, 120 genera, and 206 species were recorded in the six secondary forests at the USJ. Fifty families and 136 species were identified in the 12-year-old areas, and 57 families and 161 species were encountered in the 20 -year-old areas (Table 1), which represented an approximately $15 \%$ increase in the number of species. Of the 206 species encountered, 115 were trees $(56 \%)$, 48 shrubs (23\%), 16 herbs (8\%), 24 woody lianas (12\%), and three were epiphytes $(1.5 \%)$ (Table 2$)$. In spite of the fact that there was a $20 \%$ increase in the number of arboreal species in the 20 -year-old

Table 1. Species recorded in six secondary forests (three 12-year and three 20-year old regeneration forests) and mature forests (Silva 2004, Rocha et al. 2008, Silva et al. 2008) in Usina São José, Igarassu municipality.

\begin{tabular}{|c|c|c|c|c|}
\hline Species & Growth form & 12 years & 20 years & Mature \\
\hline \multicolumn{5}{|l|}{ Alliaceae } \\
\hline Hippeastrum stylosum Herb. & herb & & $\mathrm{x}$ & \\
\hline \multicolumn{5}{|l|}{ Anacardiaceae } \\
\hline Mangifera indica $\mathrm{L}$. & tree & & $\mathrm{x}$ & $\mathrm{x}$ \\
\hline Tapirira guianensis Aubl. & tree & $\mathrm{x}$ & $\mathrm{x}$ & $\mathrm{x}$ \\
\hline Thyrsodium spruceanum Benth. & tree & $\mathrm{x}$ & $\mathrm{x}$ & $\mathrm{x}$ \\
\hline \multicolumn{5}{|l|}{ Annonaceae } \\
\hline Anaxagorea dolichocarpa Sprague \& Sandwith & tree & $\mathrm{x}$ & $\mathrm{x}$ & $\mathrm{x}$ \\
\hline Annona montana Macfad. & tree & $\mathrm{x}$ & & \\
\hline Guatteria pogonopus Mart. & tree & & $\mathrm{x}$ & $\mathrm{x}$ \\
\hline Guatteria schomburgkiana Mart. & tree & $\mathrm{x}$ & $\mathrm{x}$ & $\mathrm{x}$ \\
\hline Guatteria sp. & tree & & $\mathrm{x}$ & \\
\hline Xylopia frutescens Aubl. & tree & $\mathrm{x}$ & $\mathrm{x}$ & $\mathrm{x}$ \\
\hline \multicolumn{5}{|l|}{ Apocynaceae } \\
\hline Allamanda cathartica $\mathrm{L}$. & woody liana & $\mathrm{x}$ & $\mathrm{x}$ & \\
\hline Allamanda sp. & woody liana & $\mathrm{x}$ & & \\
\hline Aspidospema discolor A.DC. & tree & & & $\mathrm{x}$ \\
\hline Himathanthus phagedaenicus (Mart.) Woodson & tree & $\mathrm{x}$ & $\mathrm{x}$ & $\mathrm{x}$ \\
\hline Rauvolfia grandiflora Mart. ex A.DC. & shrub & $\mathrm{x}$ & $\mathrm{x}$ & $\mathrm{x}$ \\
\hline Rauvolfia ligustrina Willd. ex Roem. \& Schult. & shrub & $\mathrm{x}$ & & \\
\hline Tabernaemontana salzmannii A.DC. & tree & & & $\mathrm{x}$ \\
\hline \multicolumn{5}{|l|}{ Araliaceae } \\
\hline Schefflera morototoni (Aubl.) Maguire, Steyerm. \& Frodim & tree & $\mathrm{x}$ & $\mathrm{x}$ & $\mathrm{x}$ \\
\hline \multicolumn{5}{|l|}{ Arecaceae } \\
\hline Acrocomia sclerocarpa Mart. & palm tree & $\mathrm{x}$ & $\mathrm{x}$ & \\
\hline Bactris ferruginea Burret & palm tree & & & $\mathrm{x}$ \\
\hline Desmoncus polyacanthos Mart. & woody liana & $\mathrm{x}$ & & \\
\hline Elaeis guineensis A.Chev. & palm tree & $\mathrm{x}$ & $\mathrm{x}$ & \\
\hline \multicolumn{5}{|l|}{ Aspleniaceae } \\
\hline Asplenium sp. & herb & $\mathrm{x}$ & & \\
\hline
\end{tabular}


Table 1. Continued...

\begin{tabular}{|c|c|c|c|c|}
\hline Species & Growth form & 12 years & 20 years & Mature \\
\hline \multicolumn{5}{|l|}{ Asteraceae } \\
\hline Chromlaena sp. & shrub & $\mathrm{x}$ & & \\
\hline Conocliniopsis prassifolia (DC.) R.M.King \& H.Rob. & shrub & $\mathrm{x}$ & & \\
\hline Conyza sumatrensis (Retz.) E. Walker & shrub & $\mathrm{x}$ & & \\
\hline Tilesia baccata (L.) Pruski & shrub & & $\mathrm{x}$ & \\
\hline Verbesina macrantha A.Rich. & shrub & $\mathrm{x}$ & & \\
\hline Vernonia brasiliana (L.) Druce & shrub & & $\mathrm{x}$ & \\
\hline \multicolumn{5}{|l|}{ Bignoniaceae } \\
\hline Adenocalymma sp. & woody liana & $\mathrm{x}$ & & \\
\hline Lundia cordata (Vell.) DC. & woody liana & & $\mathrm{x}$ & \\
\hline \multicolumn{5}{|l|}{ Boraginaceae } \\
\hline Cordia multispicata Cham. & shrub & & $\mathrm{x}$ & \\
\hline Cordia nodosa Lam. & shrub & $\mathrm{x}$ & $\mathrm{x}$ & \\
\hline Cordia polycephala (Lam.) I.M. Johnst. & tree & $\mathrm{x}$ & & \\
\hline Cordia sellowiana Cham. & tree & $\mathrm{x}$ & $\mathrm{x}$ & \\
\hline Cordia superba Cham. & tree & & $\mathrm{x}$ & $\mathrm{x}$ \\
\hline Tournefortia candidula (Miers) I.M. Johnst. & shrub & $\mathrm{x}$ & & \\
\hline Boraginaceae 1 & shrub & & $\mathrm{x}$ & \\
\hline \multicolumn{5}{|l|}{ Burseraceae } \\
\hline Protium arachouchini (Aubl.) Marchand & tree & & & $\mathrm{x}$ \\
\hline Protium giganteum Engl. & tree & & $\mathrm{x}$ & $\mathrm{x}$ \\
\hline Protium heptaphyllum L. Marchand & tree & $\mathrm{x}$ & $\mathrm{x}$ & $\mathrm{x}$ \\
\hline Tetragastris catuaba Soares da Cunha & tree & & & $\mathrm{x}$ \\
\hline \multicolumn{5}{|l|}{ Canabaceae } \\
\hline Trema micrantha (L.) Blume & tree & $\mathrm{x}$ & $\mathrm{x}$ & $\mathrm{x}$ \\
\hline \multicolumn{5}{|l|}{ Celastraceae } \\
\hline Maytenus distichophylla Mart. ex Reissek & tree & $\mathrm{x}$ & $\mathrm{x}$ & $\mathrm{x}$ \\
\hline Maytenus obtusifolia Mart. & tree & & $\mathrm{x}$ & \\
\hline \multicolumn{5}{|l|}{ Chrysobalanaceae } \\
\hline Hirtella racemosa Ruiz \& Pav. & shrub & $\mathrm{x}$ & $\mathrm{x}$ & \\
\hline \multicolumn{5}{|l|}{ Clusiaceae } \\
\hline Clusia nemorosa G.Mey. & tree & & & $\mathrm{x}$ \\
\hline Symphonia globulifera L.f. & tree & $\mathrm{x}$ & & \\
\hline Vismia guianensis (Aubl.) Pers. & tree & $\mathrm{x}$ & $\mathrm{x}$ & $\mathrm{x}$ \\
\hline \multicolumn{5}{|l|}{ Coclospermaceae } \\
\hline Cochlospermum vitifolium Spreng. & tree & & $\mathrm{x}$ & \\
\hline \multicolumn{5}{|l|}{ Convolvulaceae } \\
\hline Ipomoea alba $\mathrm{L}$. & woody liana & $\mathrm{x}$ & $\mathrm{x}$ & \\
\hline \multicolumn{5}{|l|}{ Cyperaceae } \\
\hline Cyperus rotundus $\mathrm{L}$. & herb & $\mathrm{x}$ & $\mathrm{x}$ & \\
\hline \multicolumn{5}{|l|}{ Dilleniaceae } \\
\hline Davilla aspera (Aubl.) Benoist & woody liana & $\mathrm{x}$ & & \\
\hline Doliocarpus dentatus (Aubl.) Standl. & woody liana & & $\mathrm{x}$ & \\
\hline Tetracera breyniana Schltdl. & woody liana & $\mathrm{x}$ & $\mathrm{x}$ & \\
\hline \multicolumn{5}{|l|}{ Dryopteridaceae } \\
\hline Cystopteris sp. & herb & & $\mathrm{x}$ & \\
\hline \multicolumn{5}{|l|}{ Erythroxylaceae } \\
\hline Erythroxylum citrifolium A. St.-Hil. & shrub & & $\mathrm{x}$ & $\mathrm{x}$ \\
\hline Erythroxylum mucronatum Sw. & shrub & $\mathrm{x}$ & & $\mathrm{x}$ \\
\hline \multicolumn{5}{|l|}{ Euphorbiaceae } \\
\hline Croton floribundus Spreng. & tree & & $\mathrm{x}$ & \\
\hline Pera ferruginea (schott) Mull. Arg. & tree & $\mathrm{x}$ & $\mathrm{x}$ & $\mathrm{x}$ \\
\hline Pogonophora schomburgkiana Miers ex Benth. & tree & $\mathrm{x}$ & $\mathrm{x}$ & $\mathrm{x}$ \\
\hline Sapium glandulosum (L.) Morong & tree & $\mathrm{x}$ & & \\
\hline
\end{tabular}


Table 1. Continued...

\begin{tabular}{|c|c|c|c|c|}
\hline Species & Growth form & 12 years & 20 years & Mature \\
\hline \multicolumn{5}{|l|}{ Fabaceae-Caesalpinoideae } \\
\hline Chamaecrista ensiformis (Vell.) H.S.Irwin \& Barneby & tree & & & $\mathrm{x}$ \\
\hline Apuleia leiocarpa (Vogel) J.F. Macbr. & tree & $\mathrm{x}$ & & \\
\hline Dialium guianense (Aubl.) Sandwith & tree & $\mathrm{x}$ & & $\mathrm{x}$ \\
\hline Sclerolobium densiflorum & tree & & & $\mathrm{x}$ \\
\hline Senna georgica H.S. Irwin \& Barneby & shrub & $\mathrm{x}$ & $\mathrm{x}$ & \\
\hline Senna quinquangulata (L.C.Rich.) H.S.Irwin \& Barneby & woody liana & $\mathrm{x}$ & $\mathrm{x}$ & \\
\hline Swartzia pickelii Killip ex Ducke & tree & $\mathrm{x}$ & $\mathrm{x}$ & $\mathrm{x}$ \\
\hline Fabaceae-Caesalpinoideae 1 & tree & $\mathrm{x}$ & & \\
\hline \multicolumn{5}{|l|}{ Fabaceae-Mimosoideae } \\
\hline Albizia polycephala (Benth.) Killip. & tree & $\mathrm{x}$ & $\mathrm{x}$ & \\
\hline Albizia saman (Jacq.) F. Muell. & tree & $\mathrm{x}$ & $\mathrm{x}$ & \\
\hline Inga blanchetiana Benth. & tree & & & $\mathrm{x}$ \\
\hline Inga capitata Desv. & tree & & & $\mathrm{x}$ \\
\hline Inga cayennensis Sagot ex Benth. & tree & $\mathrm{x}$ & $\mathrm{x}$ & \\
\hline Inga flagelliformis (Vell.) Mart. & tree & $\mathrm{x}$ & $\mathrm{x}$ & $\mathrm{x}$ \\
\hline Inga ingoides (Rich.) Willd. & tree & $\mathrm{x}$ & $\mathrm{x}$ & \\
\hline Inga laurina (Sw.) Willd. & tree & & $\mathrm{x}$ & \\
\hline Inga thibaudiana DC. & tree & $\mathrm{x}$ & $\mathrm{x}$ & $\mathrm{x}$ \\
\hline Inga striata Benth. & tree & & & $\mathrm{x}$ \\
\hline Parkia pendula (Willd.) Benth. ex Walp. & tree & & & $\mathrm{x}$ \\
\hline Plathymenia foliolosa Benth. & tree & & & $\mathrm{x}$ \\
\hline Plathymenia reticulata Benth. & tree & & $\mathrm{x}$ & $\mathrm{x}$ \\
\hline Fabaceae-Mimosoideae 1 & woody liana & $\mathrm{x}$ & $\mathrm{x}$ & \\
\hline Fabaceae-Mimosoideae 2 & tree & & $\mathrm{x}$ & \\
\hline \multicolumn{5}{|l|}{ Fabaceae-Papilionoideae } \\
\hline Andira fraxinifolia Benth. & tree & $\mathrm{x}$ & $\mathrm{x}$ & $\mathrm{x}$ \\
\hline Andira nitida Mart. ex Benth. & tree & $\mathrm{x}$ & & $\mathrm{x}$ \\
\hline Bauhinia microstachya (Raddi) J.F. Macbr. & shrub & & $\mathrm{x}$ & \\
\hline Bowdichia virgilioides Kunth & tree & $\mathrm{x}$ & $\mathrm{x}$ & $\mathrm{x}$ \\
\hline Desmodium axillare (Sw.) DC. & herb & & $\mathrm{x}$ & \\
\hline Dioclea virgata (L.C.Rich.) Amshoff & woody liana & & $\mathrm{x}$ & \\
\hline Dioclea sp. & woody liana & $\mathrm{x}$ & & \\
\hline Diplotropis purpurea (Rich.) Amshoff. & tree & & & $\mathrm{x}$ \\
\hline Indigofera suffruticosa Mill. & shrub & & $\mathrm{x}$ & \\
\hline Machaerium hirtum (Vell.) Stellfeld & tree & $\mathrm{x}$ & $\mathrm{x}$ & \\
\hline Machaerium salzmannii Benth. & tree & $\mathrm{x}$ & $\mathrm{x}$ & \\
\hline Phanera outimouta (Aubl.) L.P. Queiroz & woody liana & & $\mathrm{x}$ & \\
\hline Pterocarpus rohrii Vahl & tree & & $\mathrm{x}$ & \\
\hline Stylosanthes guianensis (Aubl.) Sw. & shrub & & $\mathrm{x}$ & \\
\hline Fabaceae-Papilionoideae 1 & shrub & $\mathrm{x}$ & & \\
\hline Fabaceae-Papilionoideae 2 & tree & $\mathrm{x}$ & & \\
\hline Fabaceae-Papilionoideae 3 & tree & & $\mathrm{x}$ & \\
\hline Fabaceae-Papilionoideae 4 & shrub & & $\mathrm{x}$ & \\
\hline \multicolumn{5}{|l|}{ Heliconiaceae } \\
\hline Heliconia sp. & herb & $\mathrm{x}$ & $\mathrm{x}$ & \\
\hline \multicolumn{5}{|l|}{ Hernandiaceae } \\
\hline Sparattanthelium botocudorum Mart. & shrub & $\mathrm{x}$ & $\mathrm{x}$ & $\mathrm{x}$ \\
\hline \multicolumn{5}{|l|}{ Lauraceae } \\
\hline Ocotea gardneri Mez & tree & & $\mathrm{x}$ & \\
\hline Ocotea glomerata (Nees) Mez & tree & $\mathrm{x}$ & $\mathrm{x}$ & $\mathrm{x}$ \\
\hline Ocotea limae Vattimo & tree & & $\mathrm{x}$ & $\mathrm{x}$ \\
\hline \multicolumn{5}{|l|}{ Lecythidaceae } \\
\hline Lecythis pisonis & tree & & & $\mathrm{x}$ \\
\hline
\end{tabular}


Table 1. Continued...

\begin{tabular}{|c|c|c|c|c|}
\hline Species & Growth form & 12 years & 20 years & Mature \\
\hline Eschweilera ovata (Cambess.) Miers. & tree & $\mathrm{x}$ & $\mathrm{x}$ & $\mathrm{x}$ \\
\hline Gustavia augusta $\mathrm{L}$. & tree & $\mathrm{x}$ & $\mathrm{x}$ & $\mathrm{x}$ \\
\hline \multicolumn{5}{|l|}{ Loganiaceae } \\
\hline Strychnos bahiensis Krukoff \& Barneby & shrub & $\mathrm{x}$ & $\mathrm{x}$ & \\
\hline \multicolumn{5}{|l|}{ Loranthaceae } \\
\hline Struthanthus sp. 1 & shrub & & $\mathrm{x}$ & \\
\hline Struthanthus sp. 2 & shrub & & $\mathrm{x}$ & \\
\hline \multicolumn{5}{|l|}{ Malpighiaceae } \\
\hline Byrsonima sericea DC. & tree & $\mathrm{x}$ & $\mathrm{x}$ & $\mathrm{x}$ \\
\hline Malpighiaceae 1 & woody liana & & $\mathrm{x}$ & \\
\hline \multicolumn{5}{|l|}{ Malvaceae } \\
\hline Apeiba tibourbou Aubl. & tree & $\mathrm{x}$ & $\mathrm{x}$ & $\mathrm{x}$ \\
\hline Eriotheca crenulaticalyx A.Robyns & tree & & & $\mathrm{x}$ \\
\hline Guazuma ulmifolia Pers. & tree & $\mathrm{x}$ & $\mathrm{x}$ & $\mathrm{x}$ \\
\hline Luehea paniculata Mart. & tree & & $\mathrm{x}$ & $\mathrm{x}$ \\
\hline Luehea ochrophylla & tree & & & $\mathrm{x}$ \\
\hline Triumfetta semitriloba Jacq. & shrub & $\mathrm{x}$ & $\mathrm{x}$ & \\
\hline \multicolumn{5}{|l|}{ Melastomataceae } \\
\hline Clidemia capitellata (Bonpl.) D.Don. & shrub & $\mathrm{x}$ & $\mathrm{x}$ & \\
\hline Clidemia hirta Cong. & shrub & $\mathrm{x}$ & & \\
\hline Henriettea succosa (Aubl.) DC. & tree & $\mathrm{x}$ & & $\mathrm{x}$ \\
\hline Miconia albicans (Benth.) Triana & shrub & $\mathrm{x}$ & $\mathrm{x}$ & $\mathrm{x}$ \\
\hline Miconia ciliata (Rich.) DC. & shrub & $\mathrm{x}$ & $\mathrm{x}$ & $\mathrm{x}$ \\
\hline Miconia minultiflora (Bonpl.) DC. & tree & $\mathrm{x}$ & $\mathrm{x}$ & $\mathrm{x}$ \\
\hline Miconia prasina (Sw.) DC. & tree & $\mathrm{x}$ & $\mathrm{x}$ & $\mathrm{x}$ \\
\hline \multicolumn{5}{|l|}{ Meliaceae } \\
\hline Trichilia lepidota Mart. & tree & & $\mathrm{x}$ & $\mathrm{x}$ \\
\hline \multicolumn{5}{|l|}{ Monimiaceae } \\
\hline Siparuna guianensis Aubl. & tree & $\mathrm{x}$ & $\mathrm{x}$ & \\
\hline \multicolumn{5}{|l|}{ Moraceae } \\
\hline Artocarpus heterophyllus Lam & tree & $\mathrm{x}$ & $\mathrm{x}$ & $\mathrm{x}$ \\
\hline Brosimum guianense (Aubl.) Huber & tree & $\mathrm{x}$ & $\mathrm{x}$ & $\mathrm{x}$ \\
\hline Brosimum rubescens Taub. & tree & & & $\mathrm{x}$ \\
\hline Sorocea hilarii Gaudich. & tree & & $\mathrm{x}$ & $\mathrm{x}$ \\
\hline \multicolumn{5}{|l|}{ Myrsinaceae } \\
\hline Rapanea guianensis Aubl. & tree & & $\mathrm{x}$ & $\mathrm{x}$ \\
\hline \multicolumn{5}{|l|}{ Myrtaceae } \\
\hline Calyptranthes brasiliensis (Aubl.) DC. & tree & & $\mathrm{x}$ & $\mathrm{x}$ \\
\hline Campomanesia dichotoma (O. Berg.) Mattos & tree & $\mathrm{x}$ & $\mathrm{x}$ & \\
\hline Eugenia candolleana (O. Berg) Kiaersk. & tree & $\mathrm{x}$ & $\mathrm{x}$ & \\
\hline Eugenia florida DC. & tree & & $\mathrm{x}$ & \\
\hline Eugenia punicifolia (Kunth) DC. & tree & & $\mathrm{x}$ & \\
\hline Eugenia sp. & tree & $\mathrm{x}$ & $\mathrm{x}$ & \\
\hline Myrcia bergiana O. Berg. & tree & & $\mathrm{x}$ & \\
\hline Myrcia guianensis (Aubl.) DC. & tree & & $\mathrm{x}$ & $\mathrm{x}$ \\
\hline Myrcia racemosa Barb. Rodr. & tree & $\mathrm{x}$ & $\mathrm{x}$ & $\mathrm{x}$ \\
\hline Myrcia splendens (Sw.) DC. & tree & & & $\mathrm{x}$ \\
\hline Myrcia sylvatica (G. Mey.) DC. & tree & $\mathrm{x}$ & $\mathrm{x}$ & $\mathrm{x}$ \\
\hline Myrcia tomentosa (Aubl.) DC. & tree & $\mathrm{x}$ & $\mathrm{x}$ & \\
\hline Psidium araca Raddi & tree & $\mathrm{x}$ & & \\
\hline Psidium guineense $\mathrm{Sw}$. & tree & $\mathrm{x}$ & $\mathrm{x}$ & \\
\hline Psidium guajava $\mathrm{L}$. & tree & & $\mathrm{x}$ & \\
\hline \multicolumn{5}{|l|}{ Nyctaginaceae } \\
\hline Guapira laxa (Netto) Furlan & tree & $\mathrm{x}$ & $\mathrm{x}$ & $\mathrm{x}$ \\
\hline Guapira nitida (Schmidt) Lundell & tree & $\mathrm{x}$ & $\mathrm{x}$ & \\
\hline
\end{tabular}


Table 1. Continued...

\begin{tabular}{l} 
Species \\
\hline Guapira opposita (Vell.) Reitz \\
Neea sp. \\
Ochnaceae \\
Ouratea hexasperma (A.St.-Hil.) Baill. \\
Ouratea polygyna Engl.
\end{tabular}

Olacaceae

Schoepfia brasiliensis A. DC

\section{Orchidaceae}

Orchidaceae 1

Orchidaceae 2

\section{Passifloraceae}

Passiflora alata Curtis

\section{Peraceae}

Chaetocarpus myrsinites Baill.

\section{Piperaceae}

Piper arboreum Aubl.

Piper colubrinum (Link ex Kunth) Link ex C. DC.

Piper marginatum Jacq.

\section{Phyllanthaceae}

Margaritaria nobilis L.f.

\section{Poaceae}

Lasiacis sorghoidea (Ham.) Hitchc. \& Chase

Urochloa fusca (Sw.) B.F. Hansen \& Wunderlin

Poaceae 1

Poaceae 2

Poaceae 3

Poaceae 4

Poaceae 5

Polygalaceae

Bredemeyera sp.

\section{Polygonaceae}

Coccoloba laevis Casar.

Coccoloba mollis Casar.

\section{Pteridaceae}

Pellaea sp.

Rhamnaceae

Colubrina glandulosa Perkins

\section{Rubiaceae}

Alseis floribunda Schott

Alseis pickelii Pilg. \& Schmale.

Genipa americana L.

Palicourea crocea (Sw.) Roem. \& Schult.

Posoqueria sp.

Psychotria barbiflora DC.

Psychotria bracteocardia (DC.) Mull. Arg.

Psychotria capitata Ruiz \& Pav.

Psychotria carthagenensis Jacq.

Psychotria hoffmannseggiana (Willd. ex Roem. \& Schult.)

Sabicea grisea Cham. \& Schltdl.

Rubiaceae 1

Rubiaceae 2

Rubiaceae 3

Rubiaceae 4

\section{Rutaceae}

Hortia arborea Engl.

\begin{tabular}{|c|c|c|c|}
\hline Growth form & 12 years & 20 years & Mature \\
\hline tree & & $\mathrm{x}$ & $\mathrm{x}$ \\
\hline tree & & $\mathrm{x}$ & \\
\hline tree & & & $\mathrm{x}$ \\
\hline tree & $\mathrm{x}$ & & \\
\hline shrub & $\mathrm{x}$ & & \\
\hline herb & & $\mathrm{x}$ & \\
\hline herb & & $\mathrm{x}$ & \\
\hline woody liana & & $\mathrm{x}$ & \\
\hline tree & & & $\mathrm{x}$ \\
\hline shrub & $\mathrm{x}$ & $\mathrm{x}$ & \\
\hline shrub & & $\mathrm{x}$ & \\
\hline shrub & $\mathrm{x}$ & $\mathrm{x}$ & \\
\hline tree & & & $\mathrm{x}$ \\
\hline herb & & $\mathrm{x}$ & \\
\hline herb & $\mathrm{x}$ & $\mathrm{x}$ & \\
\hline herb & & $\mathrm{x}$ & \\
\hline herb & $\mathrm{x}$ & & \\
\hline herb & $\mathrm{x}$ & & \\
\hline herb & $\mathrm{x}$ & & \\
\hline herb & & $\mathrm{x}$ & \\
\hline woody liana & $\mathrm{x}$ & & \\
\hline tree & & $\mathrm{x}$ & \\
\hline tree & $\mathrm{x}$ & $\mathrm{x}$ & $\mathrm{x}$ \\
\hline herb & & $\mathrm{x}$ & \\
\hline tree & & $\mathrm{x}$ & \\
\hline tree & $\mathrm{x}$ & $\mathrm{x}$ & $\mathrm{x}$ \\
\hline tree & & & $\mathrm{x}$ \\
\hline tree & $\mathrm{x}$ & & \\
\hline shrub & $\mathrm{x}$ & $\mathrm{x}$ & \\
\hline tree & & $\mathrm{x}$ & \\
\hline shrub & $\mathrm{x}$ & $\mathrm{x}$ & \\
\hline shrub & $\mathrm{x}$ & & \\
\hline shrub & $\mathrm{x}$ & $\mathrm{x}$ & \\
\hline tree & & & $\mathrm{x}$ \\
\hline shrub & $\mathrm{x}$ & $\mathrm{x}$ & \\
\hline woody liana & & $\mathrm{x}$ & \\
\hline shrub & $\mathrm{x}$ & & \\
\hline shrub & & $\mathrm{x}$ & \\
\hline shrub & $\mathrm{x}$ & & \\
\hline shrub & & $\mathrm{x}$ & \\
\hline tree & & & $\mathrm{x}$ \\
\hline
\end{tabular}


Table 1. Continued...

\begin{tabular}{|c|c|c|c|c|}
\hline Species & Growth form & 12 years & 20 years & Mature \\
\hline Zanthoxylum rhoifolium Lam. & tree & & $\mathrm{x}$ & $\mathrm{x}$ \\
\hline \multicolumn{5}{|l|}{ Salicaceae } \\
\hline Banara brasiliensis (Schott) Benth. & tree & $\mathrm{x}$ & $\mathrm{x}$ & \\
\hline Banara guianensis Aubl. & tree & & $\mathrm{x}$ & \\
\hline Casearia arborea (Rich.) Urb. & tree & & & $\mathrm{x}$ \\
\hline Casearia hirsuta Sw. & tree & $\mathrm{x}$ & $\mathrm{x}$ & \\
\hline Casearia javitensis Kunth & tree & $\mathrm{x}$ & $\mathrm{x}$ & $\mathrm{x}$ \\
\hline Casearia sylvestris $\mathrm{Sw}$. & tree & $\mathrm{x}$ & $\mathrm{x}$ & $\mathrm{x}$ \\
\hline \multicolumn{5}{|l|}{ Sapindaceae } \\
\hline Allophylus edulis (A.St.-Hil., Cambess. \& A.Juss.) Radlk. & tree & $\mathrm{x}$ & $\mathrm{x}$ & $\mathrm{x}$ \\
\hline Cupania oblongifolia Mart. & tree & $\mathrm{x}$ & $\mathrm{x}$ & \\
\hline Cupania paniculata Cambess. & tree & $\mathrm{x}$ & $\mathrm{x}$ & \\
\hline Cupania racemosa (Vell.) Radlk. & tree & $\mathrm{x}$ & $\mathrm{x}$ & $\mathrm{x}$ \\
\hline Cupania revoluta Radlk. & tree & $\mathrm{x}$ & $\mathrm{x}$ & $\mathrm{x}$ \\
\hline Paullinia pallida $\mathrm{L}$. & woody liana & $\mathrm{x}$ & & \\
\hline Paullinia pinnata $\mathrm{L}$. & woody liana & $\mathrm{x}$ & $\mathrm{x}$ & \\
\hline Paullinia trigona Vell. & woody liana & $\mathrm{x}$ & $\mathrm{x}$ & \\
\hline Serjania salzmanniana Seem. & woody liana & & $\mathrm{x}$ & \\
\hline Talisia elephantipes Sandwith & tree & & & $\mathrm{x}$ \\
\hline Talisia esculenta (A. St.-Hil) Radlk. & tree & $\mathrm{x}$ & & \\
\hline Sapindaceae 1 & woody liana & $\mathrm{x}$ & $\mathrm{x}$ & \\
\hline \multicolumn{5}{|l|}{ Sapotaceae } \\
\hline Diploon cuspidatum (Hoehne) Cronquist & tree & & & $\mathrm{x}$ \\
\hline Pouteria bangii (Rusby) T.D.Penn. & tree & & & $\mathrm{x}$ \\
\hline Pouteria gardneri (Mart. \& Miq.) Baehni & tree & $\mathrm{x}$ & $\mathrm{x}$ & $\mathrm{x}$ \\
\hline Pouteria grandiflora (A.DC.) Baehni & tree & & & $\mathrm{x}$ \\
\hline Pouteria peduncularis (Mart. \& Eichler ex Miq.) Baehni & tree & & & $\mathrm{x}$ \\
\hline Pouteria reticulata (Engl.) Eyma & tree & & $\mathrm{x}$ & \\
\hline Pradosia lactescens (Vell.) Radlk. & tree & & & $\mathrm{x}$ \\
\hline Sapotaceae 1 & tree & $\mathrm{x}$ & $\mathrm{x}$ & \\
\hline Sapotaceae 2 & tree & & $\mathrm{x}$ & \\
\hline \multicolumn{5}{|l|}{ Scrophulariaceae } \\
\hline Scrophulariaceae 1 & shrub & $\mathrm{x}$ & & \\
\hline \multicolumn{5}{|l|}{ Simaroubaceae } \\
\hline Simarouba amara Aubl. & tree & $\mathrm{x}$ & $\mathrm{x}$ & $\mathrm{x}$ \\
\hline \multicolumn{5}{|l|}{ Smilaccaceae } \\
\hline Smilax sp. & woody liana & $\mathrm{x}$ & & \\
\hline \multicolumn{5}{|l|}{ Solanaceae } \\
\hline Solanum asperum Rich. & shrub & $\mathrm{x}$ & $\mathrm{x}$ & \\
\hline Solanum paludosum Moric. & shrub & $\mathrm{x}$ & & \\
\hline Solanum sp. & shrub & & $\mathrm{x}$ & \\
\hline Solanaceae 1 & shrub & $\mathrm{x}$ & & \\
\hline \multicolumn{5}{|l|}{ Trigoniaceae } \\
\hline Trigonia nivea Cambess. & woody liana & & $\mathrm{x}$ & \\
\hline \multicolumn{5}{|l|}{ Urticaceae } \\
\hline Cecropia pachystachya Trécul & tree & $\mathrm{x}$ & $\mathrm{x}$ & $\mathrm{x}$ \\
\hline \multicolumn{5}{|l|}{ Verbenaceae } \\
\hline Aegiphila pernambucensis Moldenke & shrub & $\mathrm{x}$ & $\mathrm{x}$ & \\
\hline Aegiphila vitelliniflora Walpers. & shrub & $\mathrm{x}$ & $\mathrm{x}$ & \\
\hline Lantana camara $\mathrm{L}$. & shrub & & $\mathrm{x}$ & \\
\hline \multicolumn{5}{|l|}{ Violaceae } \\
\hline Amphirrhox longifolia (A.St.-Hil.) Spreng. & tree & $\mathrm{x}$ & $\mathrm{x}$ & \\
\hline Paypayrola blanchetiana Tul. & tree & $\mathrm{x}$ & & $\mathrm{x}$ \\
\hline \multicolumn{5}{|l|}{ Family undetermined } \\
\hline Undetermined & tree & & $\mathrm{x}$ & \\
\hline
\end{tabular}


secondary forest, there were no significant overall differences in terms of the habits of the plants in the two regeneration ages ( $G$ test, $\mathrm{p}=0.55$, Table 2).

The NMDS analysis, applied for all arboreal species of secondary forests (12 and 20 years) and mature forests, revealed the formation of three groups, with stress of 0.093 (Figure 2). The first group was composed by the 12-year secondary forests (SF 1, 2 and 3), the second by the 20-year secondary forests (SF 4, 5 and 6), and the third, by mature forests (Silva 2004, Rocha et al. 2008, Silva et al. 2008).

When the ANOSIM was performed, the three groups previously formed by NMDS were found to be significant $(\mathrm{R}=0.983$, $\mathrm{p}=0.0036$ ), with dissimilarity (SIMPER) of $63 \%$. The two groups composed by secondary forests (SF 1-2-3 and SF4-5-6) differed in floristic composition, with dissimilarity of $57 \%$. Floristic differences were larger when 12-year secondary forest group was compared to mature forests (MF 1-2-3), with $\mathrm{R}=1$ and dissimilarity of $78 \%$ (Table 3). The high dissimilarity on floristic composition between mature and secondary forests was shaped by the presence of tree species exclusive to mature forests, such as: Aspidosperma discolor, Chamaecrista ensiformis, Parkia pendula, Inga blanchetiana, I. capitata, Pouteria bangii, P. peduncularis.

The physical and chemical parameters of soil varied significantly between the secondary forest $(p<0.05)$, regardless of age. The secondary forests differed significantly $(\mathrm{p}<0.05)$ in their soil physical and chemical parameters and the differences were not related to the forest age. SF1, SF2, and SF3 (20-years) had similar values differing from SF6 (12-years), SF4, and SF5 (Table 4).

This separation of the secondary forests independent of their times of recuperation as observed in the grouping analysis (sub-groups $\mathrm{A}$ and $\mathrm{B}$ ) was confirmed by the DCA analysis, indicating on axis $\mathrm{I}$ (eigenvalue $=0.43)$ that the concentrations of $\mathrm{K}^{+}(\mathrm{r}=0.92), \mathrm{Ca}^{2+}$ $(\mathrm{r}=0.64), \mathrm{Mg}^{2+}(\mathrm{r}=0.83)$, and the percentages of clay $(\mathrm{r}=0.89)$ and silt $(\mathrm{r}=0.96)$ were responsible for the formation of the secondary forest groups $1-2-3$, while $\mathrm{pH}(\mathrm{r}=-0.74)$ and sand $(\mathrm{r}=-0.93)$ were responsible for the formation of the secondary forest groups 4-5-6 (Table 4; Figure 3a).

Table 2. Number of families and species of different growth habits in six secondary forests (three 12-year and three 20-year old regeneration forests) in Usina São José, Igarassu municipality, Pernambuco state, Brazil (G test, $2.09, \mathrm{p}=0.55$, non-significant).

\begin{tabular}{lccccc}
\hline \multirow{2}{*}{ Habit } & \multicolumn{2}{c}{ 12 years } & & \multicolumn{2}{c}{ 20 years } \\
\cline { 2 - 3 } \cline { 5 - 6 } \cline { 5 - 6 } Tree & Families & Species & & Families & Species \\
Shrub & 31 & $78(57,4 \%)$ & & 34 & $98(60,8 \%)$ \\
Herb & 16 & $35(25,7 \%)$ & & 15 & $35(20,5 \%)$ \\
Woody liana & 9 & $7(5,1 \%)$ & & 9 & $13(8,1 \%)$ \\
Total & 50 & $16(11,8 \%)$ & & 10 & $17(10,5 \%)$ \\
\hline
\end{tabular}

Table 3. Values of R obtained in the similarity analysis (ANOSIM, upper diagonal) and percentages of dissimilarity (SIMPER, lower diagonal) between areas, using binary data of the arboreal component of of 12-year (SF1, SF2 and SF6) and 20-year secondary forests (SF3, SF4 and SF5) and mature forests $(M F 1=$ Silva (2004), MF2 = Rocha et al. (2008), MF3 = Silva et al. (2008)) in Usina São José, Igarassu, Pernambuco, Brazil.

\begin{tabular}{lccc}
\hline \multicolumn{1}{c}{ Group } & SF 1-2-3 & SF 4-5-6 & MF 1-2-3 \\
\hline SF 1-2-3 & 0 & 0.963 & 1 \\
SF 4-5-6 & $57 \%$ & 0 & 1 \\
MF 1-2-3 & $78 \%$ & $53 \%$ & 0 \\
\hline
\end{tabular}

The DCA analysis for the other plant habits indicated the formation of two groups with distinct ages (Figure $3 b$ ). On axis 1 (eigenvalues $=0.82$ ) there was a correlation of the first group (SF 1-3) with variables $\mathrm{K}^{+}(\mathrm{r}=0.57)$, clay $(\mathrm{r}=0.68)$, and silt $(\mathrm{r}=0.69)$ and of the second group (SF 2-4-5-6) with $\mathrm{pH}(\mathrm{r}=-0.97)$ and sand $(\mathrm{r}=-0.69)$, Table 4 .

\section{Discussion}

The difference in the numbers of families between the two regenerating forest ages may seem small, although the presence of families typical of mature forests (such as Aspleniaceae, Dryopteridaceae, Orchidaceae, and Pteridaceae) in the oldest sites and the increases in the numbers of species of Fabaceae, Myrtaceae, Annonaceae, and Sapotaceae indicated that there was a significant floristic enrichment over the years - even though this was not as notable when considering the plant habits.

The occurrence of a large number of species (206) in secondary forests with different times since abandonment within the same landscape, experiencing the same climate, geology and history, could be attributed to spatial heterogeneity, which allows that a high number of plant species persists due to the high resource supply (Tilman \& Pacala 1993). According to Connell (1978), different successional stages can be seen as moderately disturbed scenarios, in which disturbance occurs with moderate frequency, duration and intensity, enabling that pioneer and secondary species cohabit the same area, resulting in larger species richness, when compared to less disturbed sites (Castillo-Campos et al. 2008).

The low level of floristic similarity between the secondary and mature forests indicates that they are in distinct successional stages and corroborates with the hypothesis that the recuperation of the floristic composition of secondary forests occurs only slowly (Chazdon 2003, Chazdon et al. 2009, Piotto et al. 2009, Powers et al. 2009). However, the sharing of 68 arboreal species between the secondary and mature forests suggests that the flora of the secondary forests at the USJ tends to convergence to that of the mature forests - as has been observed

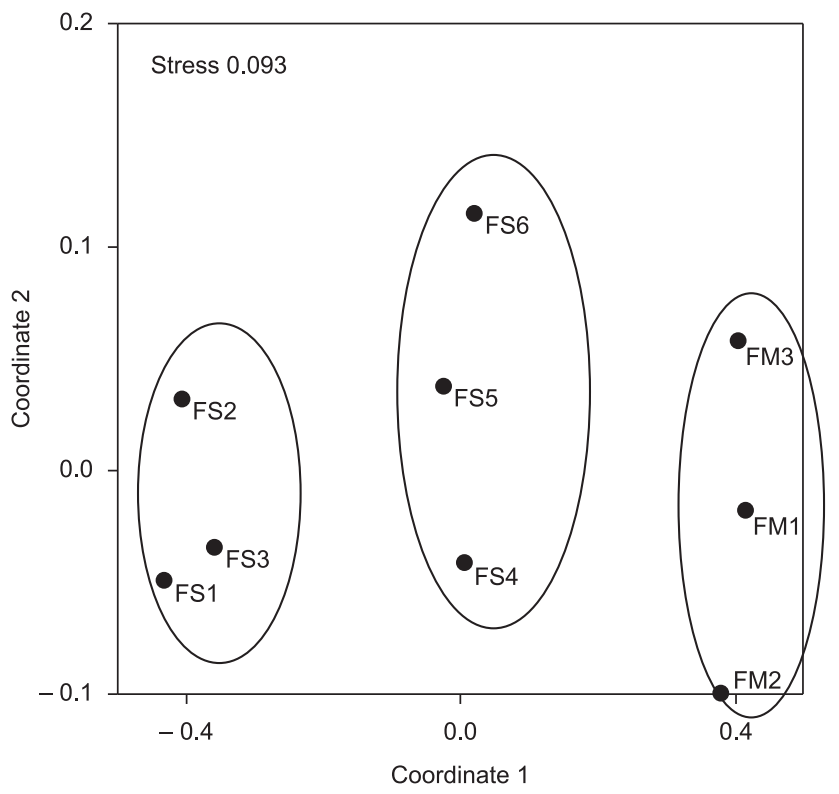

Figure 2. NMDS analysis, using the index of Jaccard, of 12-year (SF1, SF2 and SF6) and 20-year secondary forests (SF3, SF4 and SF5) and mature forests $($ MF1 = Silva (2004), MF2 = Rocha et al. (2008), MF3 = Silva et al. (2008)) in Usina São José, Igarassu municipality, Pernambuco state, Brazil. 
Nascimento, L.M. et al.

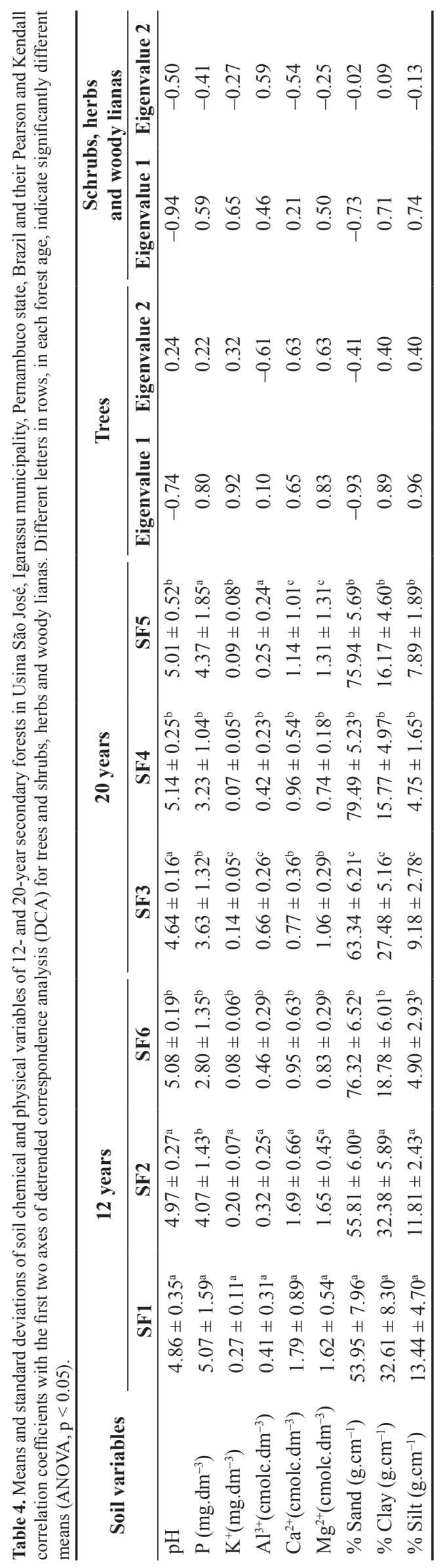




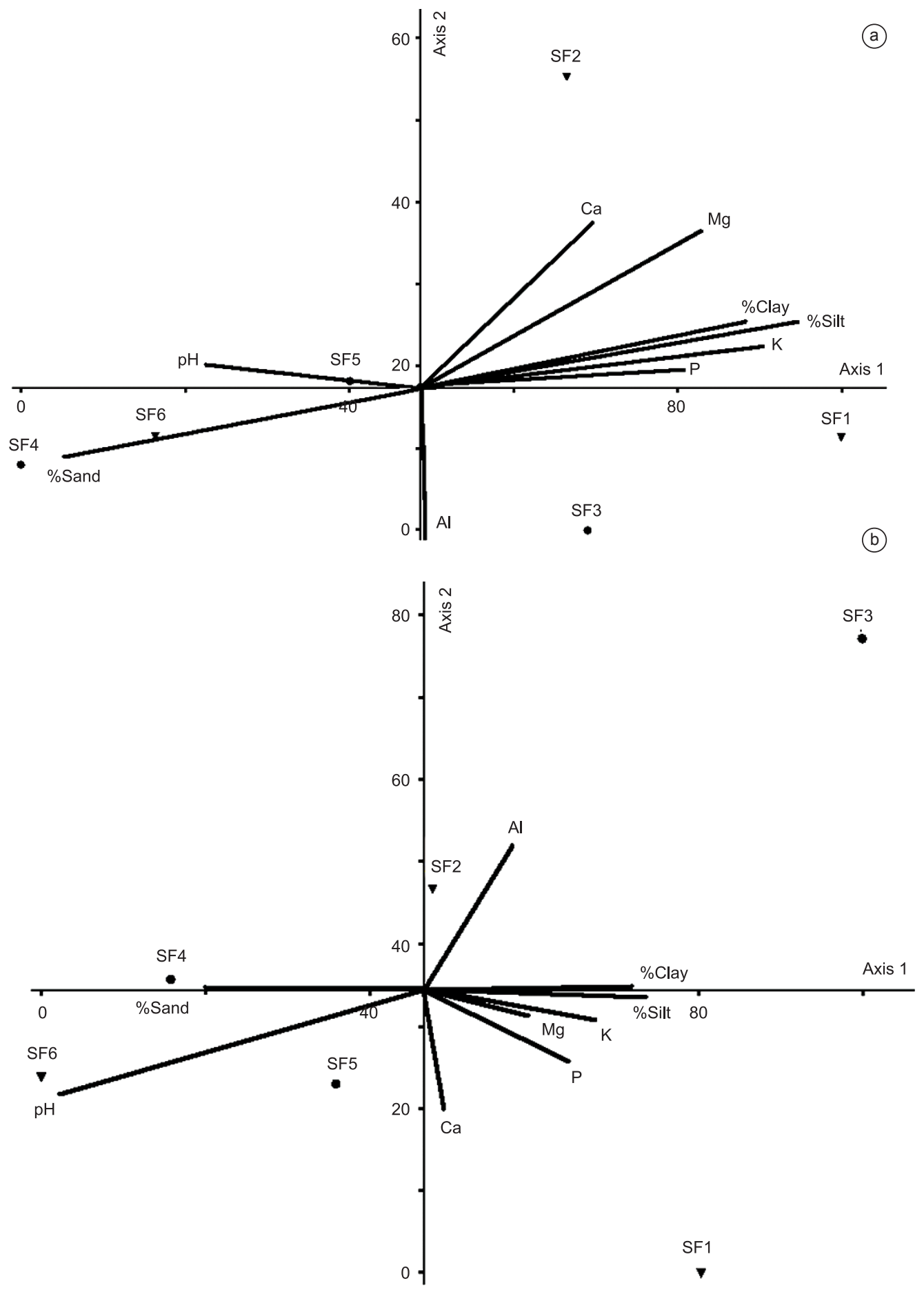

Figure 3. Ordination diagrams (DCA) of trees (a, eigenvalue $=0.43,0.12)$ and shrubs, herbs and woody lianas (b, eigenvalue $=0.82,0.24)$ of 12 -year $(\mathrm{SF} 1$, SF2 and SF6) and 20-year secondary forests (SF3, SF4 and SF5) in Usina São José, Igarassu municipality, Pernambuco state, Brazil.

in other tropical forests (Guariguata et al. 1997, Peña-Claros 2003, Capers et al. 2005, Carim et al. 2007, Chazdon et al. 2007, 2009, Liebsch et al. 2007, Castillo-Campos et al. 2008, Norden et al. 2009, Lebrija-Trejos et al. 2010).

The formation of secondary forest groups composed of distinct regeneration ages, as observed in the grouping analysis and ordinations of the arboreal habit, can be justified by the similarities of the soil characteristics within the groups (which is often a consequence of the close proximity of these areas). In terms of the other plant habits, there is also a high correlation within the groups that were formed, principally due to the similarities of soil textures (with the exception of SF 2).

All of these results indicate that not just recuperation times, but many other environmental variables influence the velocity and direction of the formation of floristic composition during secondary succession. Our results corroborate those reported for secondary forests in Costa Rica (Chazdon et al. 2009), Mexico (Powers et al. 2009), and the Brazilian Amazon (Prata et al. 2010) - which also 
did not encounter any significant correlation between the forest ages and their floristic compositions - with each locality following a distinct and idiosyncratic path of species accumulation driven by edaphic factors (Guariguata \& Ostertag 2001), colonizing species (Chazdon 2003, Junqueira et al. 2010), and the landscape matrix (Bierregaard et al. 1992, Nascimento \& Laurance 2006). Together these results indicate that the preservation of conserved forest fragments near areas undergoing regeneration permits genetic flux and the continuity of successional processes.

\section{Acknowledgements}

The authors would like to thank the Usina São José/Grupo Cavalcanti Petribú for allowing us to undertake this research on their property as well as the research groups at the Laboratório de Ecologia Vegetal (LEVE) and the Laboratório de Fitossociologia (LAFIT) of the Universidade Federal Rural de Pernambuco for their assistance with the fieldwork. This study was part of the Fragments Project, in the Brazilian-Germany Cooperation, Science and Technology for the Atlantic Forest (Proc. 690147/01-5) financed by CNPq (Conselho Nacional de Desenvolvimento Científico e Tecnológico, Brasil) and BMBF (01 LB 0203 A1), with a Doctorate CNPq grant to the first author.

\section{References}

AIDE, T.M., ZIMMERMAN, J.K., PASCARELLA, J.B., RIVERA, L. \& MARCANO-VEJA, H. 2000. Forest regeneration in a chronosequence of tropical abandoned pastures: Implications for restoration ecology. Rest. Ecol. 8:328-338. http://dx.doi.org/10.1046/j.1526-100x.2000.80048.x

ANGIOSPERM PHYLOGENY GROUP - APG III. 2009. An update of the Angiosperm Phylogeny Group classification for the orders and families of flowering plants: APG III. Bot. J. Linn. Soc. 161:105-121. http://dx.doi. org/10.1111/j.1095-8339.2009.00996.x

BIERREGAARD, R.O., LOVEJOY, T.E., KAPOS, V., SANTOS, A.A. \& HUTCHINGS, R.W. 1992. The biological dynamics of tropical rain forest fragments. Bioscience 42:859-866. http://dx.doi.org/10.2307/1312085

BROWN, S. \& LUGO, A.E. 1990. Tropical secondary forests. J. Trop. Ecol. 6:1-32. http://dx.doi.org/10.1017/S0266467400003989

CALVO, L., TÁRREGA, R. \& DE LUIS, E. 2002. Secondary succession after perturbations in a shrubland community. Acta Oecol. 23:93-404. http:// dx.doi.org/10.1016/S1146-609X(02)01164-5

CAPERS, R.S., CHAZDON, R.L., BRENES, A.R. \& ALVARADO, B.V. 2005. Successional dynamics of woody seedling communities in wet tropical secondary forests. J. Ecol. 93:1071-1084. http://dx.doi. $\operatorname{org} / 10.1111 /$ j.1365-2745.2005.01050.x

CARIM, S., SCHWARTZ, G. \& SILVA, M.F.F. 2007. Riqueza de espécies, estrutura e composição florística de uma floresta secundária de 40 anos no leste da Amazônia. Acta Bot. Bras. 21:293-308. http://dx.doi.org/10.1590/ S0102-33062007000200005

CASTILLO-CAMPOS, G., HALFFTER, G. \& MORENO, C.E. 2008. Primary and secondary vegetation patches as contributors to floristic diversity in a tropical deciduous forest landscape. Biodivers. Conserv. 17:1701-1714. http://dx.doi.org/10.1007/s10531-008-9375-7

CHAZDON, R.L. \& COE, F.G. 1999. Ethnobotany of woody species in second-growth, old-growth, and selectively logged forests of northeastern Costa Rica. Conserv. Biol. 13:1312-1322. http://dx.doi.org/10.1046/ j.1523-1739.1999.98352.x

CHAZDON, R.L. 1998. Tropical forests-log 'in or leave 'in? Science 281:1295-1296. http://dx.doi.org/10.1126/science.281.5381.1295

CHAZDON, R.L. 2003. Tropical forest recovery: legacies of human impact and natural disturbances. Perspect. Plant Ecol. 6:51-71. http://dx.doi. org/10.1078/1433-8319-00042
CHAZDON, R.L., LETCHER, S.G., VAN BREUGEL, M., MARTÍNEZRAMOS, M., BONGERS, F. \& FINEGAN, B. 2007. Rates of change in tree communities of secondary Neotropical forests following major disturbances. Phil. Trans. R. Soc. 362:273-289. http://dx.doi.org/10.1098/ rstb.2006.1990

CHAZDON, R.L., PERES, C.A., DENT, D., SHEIL, D., LUGO, A.E., LAMB, D., STORK, N.E. \& MILLER, S.E., 2009. The potential for species conservation in tropical secondary forests. Conserv. Biol. 23:1406-1417. http://dx.doi.org/10.1111/j.1523-1739.2009.01338.x

CLARKE, K.R. 1993. Non-parametric multivariate analysis of changes in community structure. Aust. J. Ecol. 18:117-143. http://dx.doi. org/10.1111/j.1442-9993.1993.tb00438.x

COHEN, J. 1988. Statistical power analysis for the behavioral sciences. Lawrence Erlbaum Associates. Hillsdale.

COMPANHIA PERNAMBUCANA DO MEIO AMBIENTE - CPRH. 2003. Diagnóstico socioambiental do litoral norte de Pernambuco. CPRH, Recife.

CONNELL, J.H. 1978. Diversity in tropical rain forest and coral reefs. Science 199:1302-1310

DANCEY, C.P. \& REIDY, J. 2006. Estatística sem matemática para psicologia: usando SPSS para Windows. Artmed, Porto Alegre.

DEAN, W. 2002. A ferro e fogo: a história e a devastação da Mata Atlântica brasileira. Cia das Letras, São Paulo.

DENSLOW, J.S. \& GUZMAN, S. 2000. Variation in stand structure, light and seedling abundance across a tropical moist forest chronosequence, Panama. J. Veg. Sci. 11:201-212. http://dx.doi.org/10.2307/3236800

DENT, D.H. \& WRIGHT, S.J. 2009. The future of tropical species in secondary forests: a quantitative review. Biol. Conserv. 142: 2833-2843. http:// dx.doi.org/10.1016/j.biocon.2009.05.035

EMBRAPA. 1997. Manual de métodos de análises de solo. Ministério da Agricultura e do Abastecimento, Rio de Janeiro.

FELDPAUSCH, T.R., PRATES-CLARK, C.C., FERNANDES, E.C.M. \& RIHA, S.J. 2007. Secondary forest growth deviation from chronosequence predictions in central Amazonia. Glob. Change Biol. 13:967-979. http:// dx.doi.org/10.1111/j.1365-2486.2007.01344.x

FELDPAUSCH, T.R., RIHA, S., FERNANDES, E.C.M. \& WANDELLI, E.V. 2005. Development of forest structure and leaf area in secondary forests regenerating on abandoned pastures in Central Amazonia. Earth Interact. 9:1-22. http://dx.doi.org/10.1175/EI140.1

FILGUEIRAS, T.S., BROCHADO, A.L., NOGUEIRA, P.E. \& GUALA II, G.F. 1994. Caminhamento: um método expedito para levantamentos florísticos qualitativos. Cad. Geoc. 12:39-43.

FINEGAN, B. \& NASI, R. 2004. The biodiversity and conservation potential of shifting cultivation landscapes. In Agroforestry and biodiversity conservation in tropical landscapes (G. Schroth, G.A.B. Fonseca, C.A. Harvey, C. Gascon, H.L. Vasconcelos \& A.M.N. Izac, eds.). Island Press, Washington, p.153-197.

FINEGAN, B. 1996. Pattern and process in neotropical secondary rain forests: the first 100 years of succession. Trends. Ecol. Evol. 11:119-124. http:// dx.doi.org/10.1016/0169-5347(96)81090-1

GEHRING, C., PARK, S. \& DENICH, M. 2004. Liana allometric biomass equations for Amazonian primary and secondary forest. For. Ecol. Manage. 195:69-83. http://dx.doi.org/10.1016/j.foreco.2004.02.054

GEIST, H.J. \& LAMBIN, E.F. 2001. What drives tropical deforestation? A meta-analysis of proximate and underlying causes based on sub-national case study evidence. CIACO, LUCC Report Series n.4. Louvain-laNeuve.

GRACE, J. 2004. Understanding and managing the global carbon cycle. J. Ecol. 92:189-202. http://dx.doi.org/10.1111/j.0022-0477.2004.00874.x

GUARIGUATA, M.R., CHAZDON, R.L., DENSLOW, J.S, DUPUY, J.M. \& ANDERSON, L. 1997. Structure and floristic of secondary and oldgrowth forest stands in lowland Costa Rica. Plant Ecol. 132:107-120. http://dx.doi.org/10.1023/A:1009726421352

GUARIGUATA, M.R. \& OSTERTAG, R. 2001. Neotropical secondary forest succession: changes in structural and functional characteristics. For. Ecol. Manage. 148:185-206. http://dx.doi.org/10.1016/S0378-1127(00)00535-1 
HAMMER, Ø., HARPER, D.A.T. \& RYAN, P.D. 2001. PAST: Paleontologica Statistics Software Package for Education and Data Analysis. Paleont Electronica 4: 9p. http://www.paleoelectronica.org/2001_1/past/ issue1_01.htm.

HERRERA-MONTES, A. \& BROKAW, N. 2010. Conservation value of tropical secondary forest: A herpetofaunal perspective. Biol. Conserv. 143:1414-1422. http://dx.doi.org/10.1016/j.biocon.2010.03.016

INTERNATIONAL TROPICAL TIMBER ORGANIZATION - ITTO. 2002. ITTO guidelines for the restoration, management and rehabilitation of degraded and secondary tropical forests. International Tropical Timber Organization, Yokohama.

JUNQUEIRA, A.B., SHEPARD JUNIOR, G.H. \& CLEMENT, C.R. 2010 Secondary forests on anthropogenic soils in Brazilian Amazonia conserve agrobiodiversity. Biodivers Conserv. 19:1933-1961. http://dx.doi. org/10.1007/s10531-010-9813-1

KÖPPEN, W. 1936. Das geographische system der klimate. Handbuch der Klimatologie, Band 5, Teil C. Gebrüder Bornträger, Berlin.

LEBRIJA-TREJOS, E., MEAVE, J.A., POORTER, L., PÉREZ-GARCÍA, E.A. \& BONGERS, F. 2010. Pathways, mechanisms and predictability of vegetation change during tropical dry forest succession. Perspect. Plant. Ecol. 12:267-275. http://dx.doi.org/10.1016/j.ppees.2010.09.002

LEGENDRE, P. \& LEGENDRE, L. 1998. Numerical Ecology. 2nd ed. Elsevier, Amsterdam.

LETCHER, S.G. \& CHAZDON, R.L. 2009. Rapid recovery of biomass, species richness, and species composition in a forest chronosequence in Northeastern Costa Rica. Biotropica 41:608-617. http://dx.doi. org/10.1111/j.1744-7429.2009.00517.x

LIEBSCH, D., GOLDENBERG, R. \& MARQUES, M.C.M. 2007. Florística e estrutura de comunidades vegetais em uma cronoseqüência de Floresta Atlântica no Paraná. Acta Bot. Bras. 21:983-992. http://dx.doi org/10.1590/S0102-33062007000400023

LIEBSCH, D., MARQUES, M.C.M. \& GOLDENBERG, R. 2008. How long does the Atlantic Rain Forest take to recover after a disturbance? Changes in species composition and ecological features during secondary succession. Biol. Conserv. 141:1717-1725. http://dx.doi.org/10.1016/j. biocon.2008.04.013

McCUNE, B. \& MEFFORD, M.J. 1999. PC-ORD - Multivariate Analysis of Ecological Data. version 4.0. MjM Software. Gleneden Beach, Oregon.

MORI, S.A., SILVA, L.A.M., LISBOA, G. \& CORADIN, L. 1989. Manual de manejo do herbário fanerogâmico. Centro de Pesquisas do Cacau, Ilhéus.

NASCIMENTO, H.E.M. \& LAURANCE, W.F. 2006. Area and edge effects on forest structure in Amazonian forest fragments after 13-17 years of isolation. Acta Amazon. 36:183-192. http://dx.doi.org/10.1590/S004459672006000200008

NORDEN, N., CHAZDON, R.L., CHAO, A., YI-HUEI JIANG, Y. \& VÍLCHEZ-ALVARADO, B. 2009. Resilience of tropical rain forests: tree community reassembly in secondary forests. Ecol. Letters 12:385-394. http://dx.doi.org/10.1111/j.1461-0248.2009.01292.x

PARRY, L., BARLOW, J. \& PERES, C.A. 2007. The conservation value of secondary forests for large vertebrates in the Brazilian Amazon. J. Trop. Ecol. 23:653-662.

PEÑA-CLAROS, M. 2003. Changes in forest structure and species composition during secondary forest succession in the Bolivian Amazon. Biotropica 35:450-461.

PIOTTO, D., MONTAGNINI, F., THOMAS, W., ASHTON, M. \& OLIVER, C. 2009. Forest recovery after swidden cultivation across a 40-year chronosequence in the Atlantic forest of southern Bahia, Brazil. Plant Ecol. 205:261-272.

POWERS, J.S., BECKNELL, J.M., IRVING, J. \& PÈREZ-AVILES, D. 2009. Diversity and structure of regenerating tropical dry forests in Costa Rica: Geographic patterns and environmental drivers. For. Ecol. Manage. 258:959-970. http://dx.doi.org/10.1016/j.foreco.2008.10.036
PRATA, S.S., MIRANDA, I.S., ALVES, S.A.O., FARIAS, F.C., JARDIM, F.C.S. 2010. Floristic gradient of the northeast paraense secondary forests. Acta Amazon. 40:523-534. http://dx.doi.org/10.1590/S004459672010000300011

PRIMACK, R.B. 2008. A primer of conservation biology. Sinauer Associates, Sunderland.

RANTA, P., BLON, T., NIEMELÃ, J., JOENSUU, E. \& SIITONEN, M. 1998. The fragmented Atlantic rain forest of Brazil: size, shape and distribution of forest fragments. Biodivers. Conserv. 7:385-403. http:/ dx.doi.org/10.1023/A:1008885813543

RIBAS, R.F., MEIRA NETO, J.A.A., SILVA, A.F., SOUZA, A.L. 2003. Composição florística de dois trechos em diferentes etapas seriais de uma floresta estacional semidecidual em Viçosa, Minas Gerais. Rev. Arvore 27:821-830. http://dx.doi.org/10.1590/S010067622003000600008

RIBEIRO, M.C., METZGER, J.P., MARTENSEN, A.C., PONZONI, F.J. \& HIROTA, M.M. 2009. The Brazilian Atlantic Forest: how much is left, and how is the remaining forest distributed? Implications for conservation. Biol. Conserv. 142:1141-1153. http://dx.doi.org/10.1016/j. biocon.2009.02.021

ROCHA, K.D, CHAVES, L.F.C., MARANGON, L.C. \& LINS E SILVA, A.C.B. 2008. Caracterização da vegetação arbórea adulta em um fragmento de floresta atlântica, Igarassu, PE. Rev. Bras. Cien. Agra. 3:35-41.

SCHESSL, M., DA SILVA, W.L. \& GOTTSBERGER, G. 2008. Effects of fragmentation on forest structure and litter dynamics in Atlantic rainforest in Pernambuco, Brazil. Flora. 203:215-228. http://dx.doi.org/10.1016/j. flora.2007.03.004

SILVA, A.G. 2004. Fisionomia e estrutura da comunidade arbórea, na Mata dos Macacos Município de Igarassu-PE. Dissertação de Mestrado. Universidade Federal Rural de Pernambuco, Recife.

SILVA, H.C.H., LINS E SILVA, A.C.B., GOMES, J.S. \& RODAL, M.J.N. 2008. The effect of internal and external edges on vegetation physiognomy and structure in a remnant of Atlantic Lowland Rainforest in Brazil. Bioremed. Biodivers. Bioavail. 2:47-55.

SMith, A.R., PRYER, K.M., SCHUETTPELZ, E., KORAll, P., SCHNEIDER, H. \& WOLF, P.G. 2008. Fern classification. In The Biology and Evolution of Ferns and Lycophytes (T.A. Ranker \& C.H. Haufler, eds.). Cambridge Univ. Press, Cambridge, p.417-467. http:// dx.doi.org/10.1017/CBO9780511541827.017

SMITH, A.R. \& WOLF, P.G. 2006. A classification for extant ferns Taxon 55:705-731. http://dx.doi.org/10.2307/25065646

SOKAL, R.R. \& ROHLF, F.J. 1995. Biometry: the principles and practices of statistics in biological research. New York: Freeman.

STATSOFT. 2001. STATISTICA. version 6.0. Stat Soft Inc., Tulsa.

TABARELLI, M. \& GASCON, C. 2005. Lessons from fragmentation research: improving management and policy guidelines for biodiversity conservation. Conserv. Biol. 19:734-739. http://dx.doi.org/10.1111/ j.1523-1739.2005.00698.x

TILMAN, D. \& PACALA, S. 1993. The maintenance of species richness in plant communities. In Species diversity in ecological communities (R.E. Ricklefs \& D. Schluter, eds.). University of Chicago Press, Chicago, p.13-25.

TRINDADE, M.B., LINS E SILVA, A.C.B., SILVA, H.P., FILGUEIRA, S.B. $\&$ SCHESSL, M. 2008. Fragmentation of the Atlantic rainforest in the Northern Coastal Region of Pernambuco, Brazil: recent changes and implications for conservation. Bioremed. Biodivers. Bioavail. 2:5-13.

VELOSO, H.P., RANGEL-FILHO, A.L.R. \& LIMA, J.C.A. 1991. Classificação da vegetação brasileira, adaptada a um sistema universal. IBGE, Rio de Janeiro.

WRIGHT, S.J. \& MULLER-LANDAU, H.C. 2006. The future of tropical forest species. Biotropica 38:287-301. http://dx.doi.org/10.1111/j.17447429.2006.00154.x 